\title{
The Effect of Intravenous Methylprednisolone on Recurrent Exacerbation in Hematologic Malignancy-associated Progressive Multifocal Leukoencephalopathy
}

\author{
Ryutaro Nakamura, Akihiro Kitamura, Takahito Tsukamoto, Ryota Tamura, Nobuhiro Ogawa, \\ Isamu Yamakawa, Hyoh Kim, Michihiro Kawai, Mitsuru Sanada and Makoto Urushitani
}

\begin{abstract}
:
We herein report a 65-year-old man with progressive multifocal leukoencephalopathy (PML) after 2-year remission from acute myeloid leukemia who developed recurrent episodes of left hemiparesis with gadolinium enhancement on magnetic resonance imaging. Steroid pulse therapy for each exacerbation induced clinical and radiological improvement, suggesting that exacerbations are an excessive immune response to the JC virus and distinct from immune reconstitution inflammatory syndrome (IRIS). Although glucocorticoids are recommended only for IRIS, steroid pulse therapy should be considered as a therapeutic option in cases of exacerbation of hematologic malignancy-associated PML. Importantly, neuroimaging is not sufficient to differentiate excessive inflammation from a controlled inflammatory response, for which steroids are not recommended.
\end{abstract}

Key words: PML, hematologic malignancy-associated PML, IRIS, inflammatory PML, PML with controlled inflammatory response

(Intern Med 60: 1287-1291, 2021)

(DOI: 10.2169/internalmedicine.5917-20)

\section{Introduction}

Progressive multifocal leukoencephalopathy (PML) is a fatal demyelinating disease of the central nervous system (CNS) that occurs almost exclusively in immunosuppressed individuals caused by reactivation of the $\mathrm{JC}$ virus $(\mathrm{JCV})$ in oligodendrocytes. PML develops in patients with human immunodeficiency virus (HIV) infection (30-40\%), those receiving immunosuppressive drugs, and those afflicted with hematologic malignancies (30-40\%) (1).

Recently, the number of side effects of immunosuppressive therapies has been increasing, particularly in cases of multiple sclerosis (MS) treated with natalizumab (2). As there is no specific treatment for PML, which has a high mortality rate, the main approach is restoring the host adaptive immune response for patients with HIV- and drugassociated PML. For patients with HIV infection, it is recommended to initiate antiretroviral therapy (ART); and for patients on immunosuppressive drugs, including natalizumab, drug discontinuation or plasma exchange is necessary $(3,4)$. The successful restoration of immunity is often accompanied by immune reconstitution inflammatory syndrome (IRIS), for which glucocorticoids are recommended. Of note, there is no standard protocol for restoring the immune response to JCV in cases of hematologic malignancyassociated PML, so the prognosis is poor (5). For bonemarrow transplant recipients who develop PML, the median length of survival is less than two years (6).

Typically, brain magnetic resonance imaging (MRI) of PML does not show mass effect, edema, or contrast enhancement, indicating the absence of inflammation in the brain (7). However, PML with a preserved immune response to JCV in the CNS demonstrates contrast enhancement on MRI, which reflects the infiltration of inflammatory cells into the brain. PML-IRIS shows marked contrast enhancement on MRI and infiltration by macrophages and CD8+ T lymphocytes on brain specimen, which occur in parallel 
with clinical worsening, for which glucocorticoids are recommended in the guidelines $(4,8,9)$. However, PML with a controlled anti-viral inflammatory response shows mild contrast enhancement on MRI and balanced infiltration of CD8+ and CD4+ T lymphocytes on brain specimens (10), for which glucocorticoids are not used due to concerns about the proliferation of JCV by suppressing the anti-viral inflammatory response proposed in a previous case report (10). It is often challenging to distinguish a controlled anti-viral inflammatory response from IRIS using neuroimaging alone, hampering the decision as to whether or not glucocorticoids should be initiated.

We herein report a case of hematologic malignancyassociated PML with inflammation in the CNS two years after bone-marrow transplantation in which intermittent steroid pulse therapy was quite effective, leading to a good prognosis.

\section{Case Report}

A 61-year-old right-handed man was admitted to our hospital for dysarthria and left hemiparesis starting that morning. The patient had undergone allogeneic hematopoietic stem cell transplantation (HSCT) for acute myeloid leukemia (AML) two years earlier. His medical history included graftversus-host disease (GVHD) and cytomegalovirus (CMV) retinitis, which were completely resolved. He had not received any immunosuppressive regimen for a year and a half.

On admission, he showed left hemispatial neglect, conjugate eye deviation to the left, dysarthria, left ataxic hemiparesis, and myoclonus in the left upper and lower limbs but no fever. Plain head MRI showed a high signal in the cortex and white matter of the right temporal lobe on diffusionweighted imaging (DWI), fluid-attenuated inversion-recovery (FLAIR) imaging, and apparent diffusion coefficient (ADC) imaging. In the cerebrospinal fluid (CSF), the protein level was mildly elevated (up to $51 \mathrm{mg} / \mathrm{dL}$ ), but the number of cells did not increase, and the glucose level was normal (71 $\mathrm{mg} / \mathrm{dL}$ ).

We suspected viral encephalitis, including herpes simplex virus or autoimmune encephalitis, and started treatment with acyclovir and steroid pulse for three days. On the second day of admission, the clinical symptoms dramatically improved with a mild residual of left hemispatial neglect and ataxic hemiparesis. Blood tests showed a decrease in the CD $4+$ cell count to $259 / \mu \mathrm{L}$. On MRI on the third day, the lesions were unchanged, but mild gadolinium enhancement at the margins of the lesion in the right temporal lobe was delineated (Fig. 1, 2). On the fourth day, JCV-DNA was reported to be positive $\left(1.45 \times 10^{4}\right.$ copies $\left./ \mathrm{mL}\right)$ in the frozen CSF sample, leading to a diagnosis of PML.

After obtaining the approval of the Ethics Committee in our hospital, we started mirtazapine at a dose of $15 \mathrm{mg} /$ day and mefloquine at a dose of $275 \mathrm{mg} /$ day for 3 days, followed by $275 \mathrm{mg}$ once per week. The clinical symptoms and radiological findings remained stable. The copy number of JCV-DNA in the CSF sample gradually decreased and became undetectable 29 days after admission. On the 35th day, however, left ataxic hemiparesis and hemispatial neglect worsened. MRI on the 36th day showed enlargement of the lesions on FLAIR with gadolinium enhancement in the right parietal lobe. Blood samples showed a recovered CD4+ cell count of $496 / \mu \mathrm{L}$, and the JCV-DNA in the CSF sample was positive again $\left(1.6 \times 10^{2}\right.$ copies $\left./ \mathrm{mL}\right)$.

We started steroid pulse therapy for three days, and the symptoms improved the next day. The dose of mirtazapine was subsequently increased to $22.5 \mathrm{mg} /$ day, and risperidone was started at a dose of $1 \mathrm{mg} /$ day. On the 53rd day, the left ataxic hemiparesis and hemispatial neglect were exacerbated, and MRI showed enlargement of the lesions on FLAIR with gadolinium enhancement again (Fig. 2). Additional steroid pulse therapy for three days was conducted, resulting in the improvement of the symptoms the next day. On the 55th day, chest X-ray revealed bilateral ground-glass appearance, and a close examination led to a diagnosis of pneumocystis pneumonia, which was managed by SulfamethoxazoleTrimethoprim. The copy number of JCV in the CSF increased to 1,150 copies $/ \mathrm{mL}$ on the 65 th day, but the clinical symptoms were stable, and it became negative on the 79th day. There was no sign of AML relapse or comorbidity of other hematologic diseases on a bone marrow biopsy during the course. He was discharged from our hospital 117 days after admission.

\section{Discussion}

In our case, PML occurred after two years of complete remission of AML following bone marrow transplantation. Based on the MRI findings of gadolinium enhancement and the rapid response to intravenous corticosteroids, we initially suspected other diseases with an inflammatory process besides PML, such as herpes simplex viral encephalitis or autoimmune encephalitis (Fig. 2). Once the diagnosis of PML was made, as there was no standard treatment for restoring the host adaptive immune response in cases of hematologic malignancy-associated PML, mefloquine, mirtazapine and risperidone were administered to suppress JCV replication. Based on the prompt effect of steroid pulse therapy, the brain inflammation indicated by gadolinium enhancement on MRI was estimated to have been excessive enough to have caused symptoms, rather than controlled. We therefore administered steroid pulse therapies repeatedly for each bout of clinical and radiological worsening in order to control the excessive inflammation induced by the preserved immune response to JCV, ultimately successfully ameliorating the symptoms. IRIS was deemed unlikely in our case due to the lack of therapeutic opportunities linked to immune reconstitution.

A preserved immune response to JCV causes inflammation in the CNS. The controlled anti-viral inflammatory response, as indicated by mild contrast enhancement on MRI 
DWI
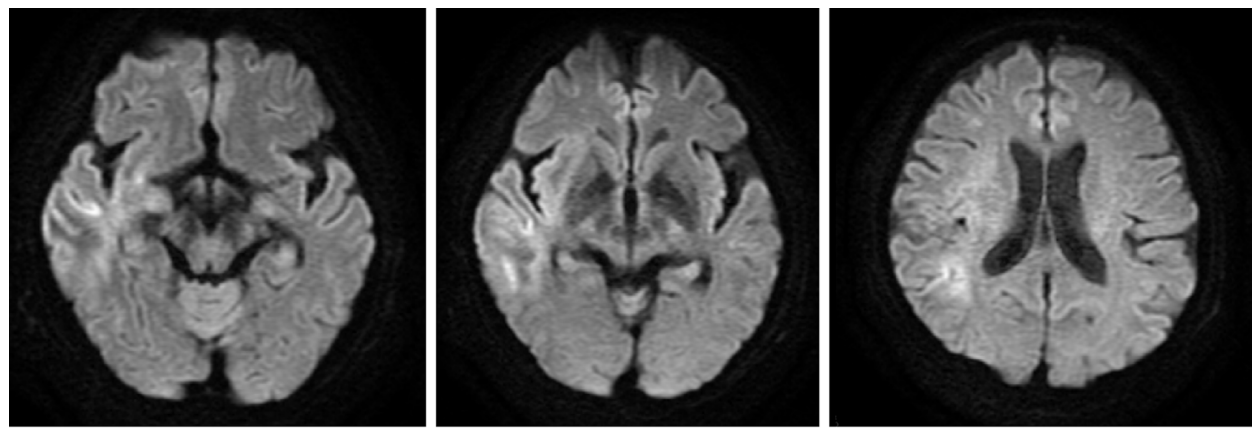

FLAIR
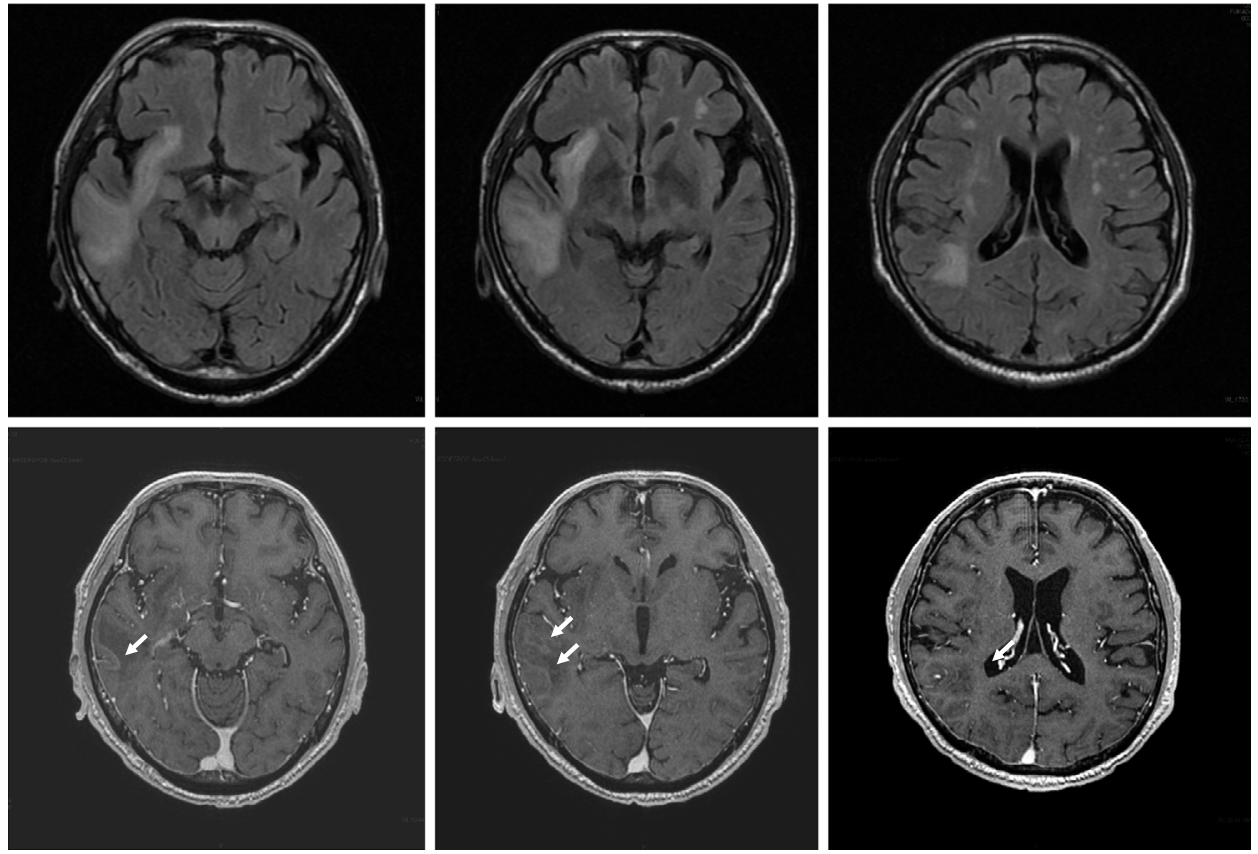

Figure 1. MRI findings on day 3 of admission during steroid pulse therapy. A high signal in the cortex and white matter of the right temporal lobe on DWI, FLAIR images with gadolinium enhancement at the margins of the lesion in the right temporal lobe (arrows). DWI: diffusion-weighted imaging, FLAIR: fluid-attenuated inversion-recovery, T1WI: T1-weighted imaging, MRI: magnetic resonance imaging, Gd: gadolinium

and balanced infiltration of CD8+ and CD4+ T lymphocytes on a brain biopsy, suggest a therapeutically desirable immune condition, which indicates a good responder to mefloquine treatment (10). However, severe inflammation in IRIS with marked contrast enhancement on MRI and infiltration by macrophages and CD8+ $\mathrm{T}$ lymphocytes is associated with clinical exacerbation (2).

Although many guidelines, including the Japanese PML Practice Guideline 2020, recommend glucocorticoid therapy for IRIS $(4,8,9)$, there is no global consensus concerning the definition of IRIS. The term IRIS originally denoted a collection of inflammatory disorders associated with a paradoxical worsening of preexisting infectious processes following the initiation of ART in HIV-infected individuals (11). At present, the term PML-IRIS is also used for patients subjected to the temporary use of immunosuppression who develop an excessive inflammatory reaction in PML lesions, namely immune reconstitution. PML-IRIS occurs mainly in patients with HIV undergoing ART and those with MS after the withdrawal of natalizumab whose immune re- sponse has been reconstituted $(2,9,12,13)$. In a previous report, patients with contrast enhancement of PML lesions on neuroimaging at the time of the diagnosis before withdrawal/removal of natalizumab were classified as "earlyPML-IRIS," which might have included patients with inflammatory PML or PML with a controlled inflammatory response. Some patients in the "early-PML-IRIS" group had a good response to steroid therapy (9). Regarding steroid treatment, the combination of early and prolonged treatment is recommended in patients with $\mathrm{HIV}$-associated PMLIRIS (8). Longer-term aggressive glucocorticoid therapy, such as repeated courses of high-dose intravenous corticoids followed by tapered doses of oral steroids, may also be necessary for natalizumab-associated PML-IRIS, which persists for at least several months (4) (Fig. 3).

However, cases of PML with a controlled anti-viral inflammatory response might have a good response to risperidone and mefloquine, which suppress JCV replication, and have a good prognosis without using glucocorticoids (10). When the inflammation is under control, glucocorticoids 


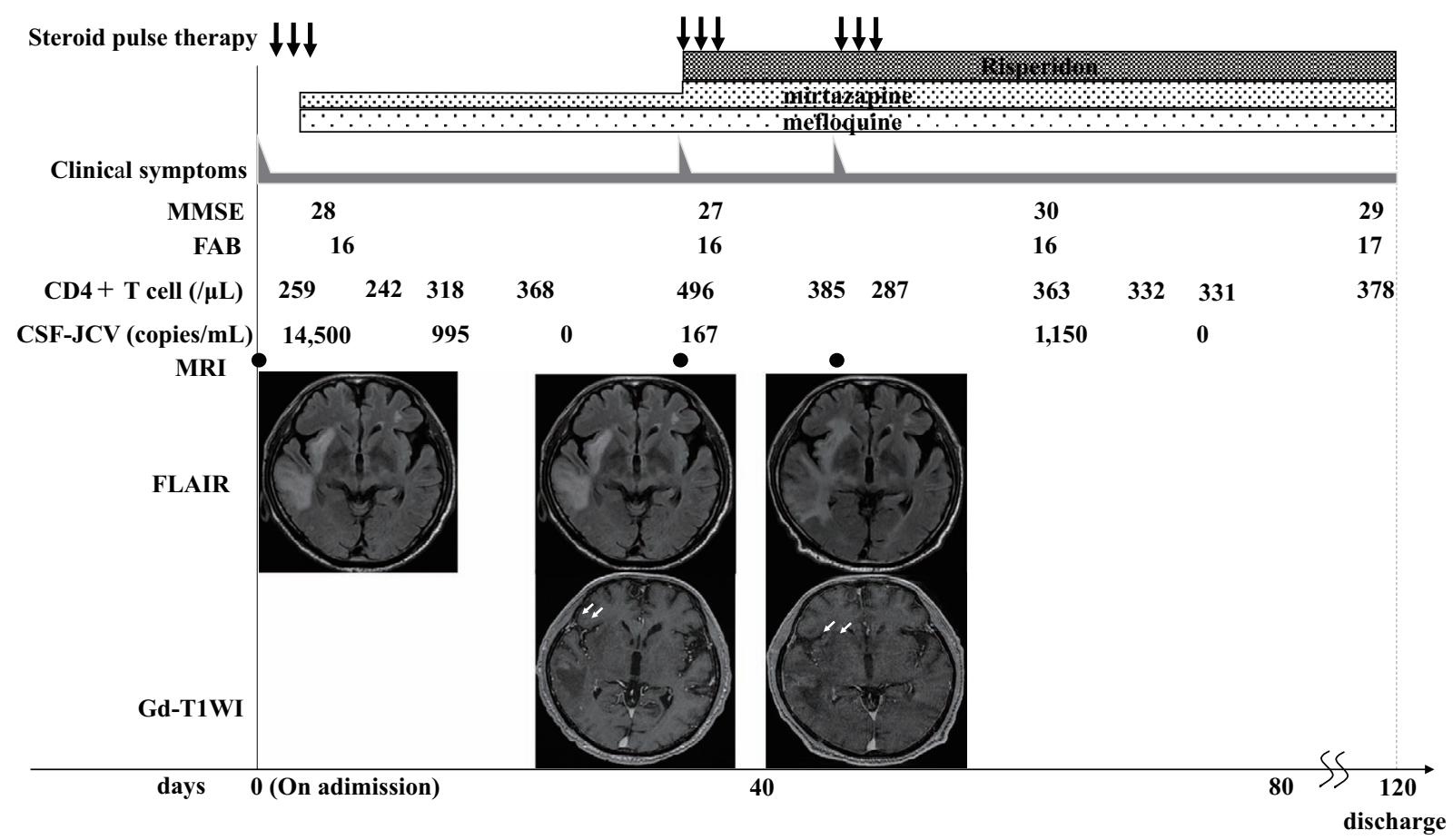

Figure 2. Clinical course. Temporal changes in the clinical symptoms, the number of CD4+T cells and DNA copies of JCV in the CSF, and MRI findings. MRI conducted when clinical worsening occurred showed lesions with gadolinium enhancement (arrows). CSF: cerebral spinal fluid, FLAIR: fluid-attenuated inversion recovery, JCV: JC virus, PML: progressive multifocal leukoencephalopathy, T1WI: T1-weighted imaging, MRI: magnetic resonance imaging, Gd: gadolinium

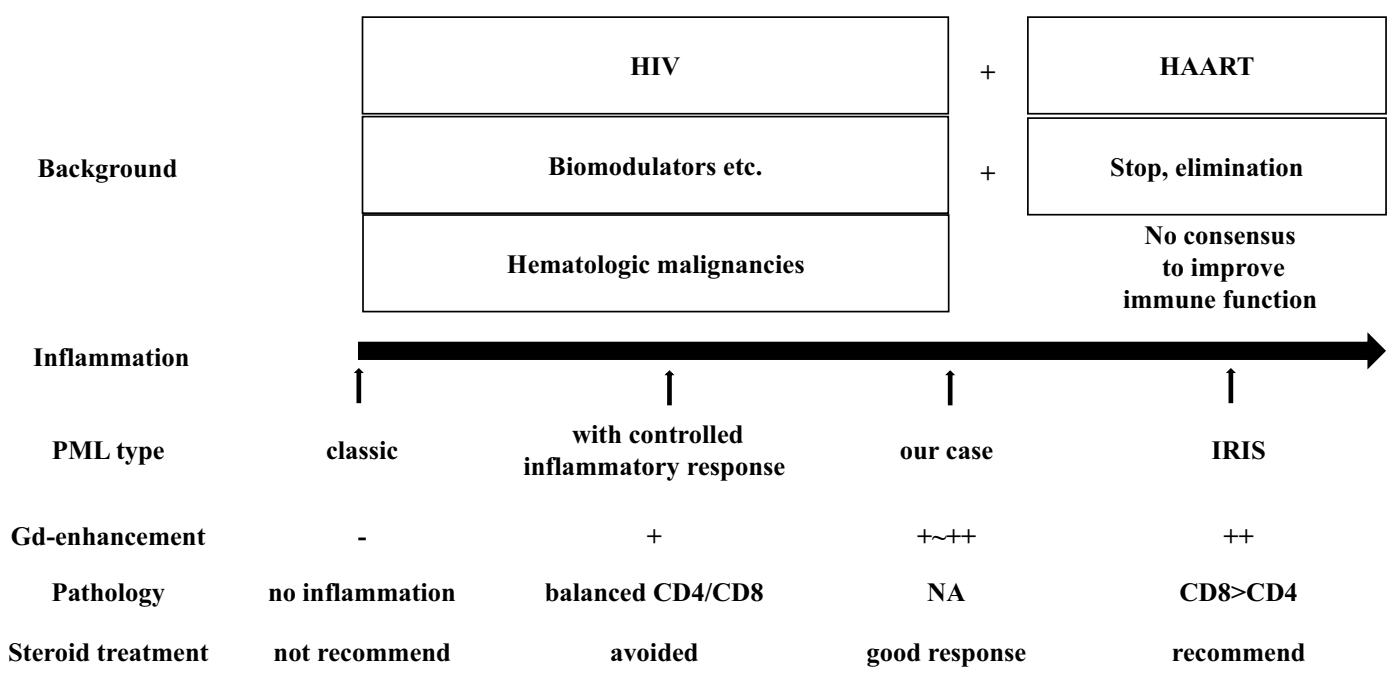

Figure 3. Classification of PML types. PML was classified according to the extent of inflammation in the CNS as classic PML, PML with a controlled inflammatory response and PML-IRIS; our case was considered to fall between the latter two types. HAART: highly active antiretroviral therapy, HIV: human immunodeficiency virus, IRIS: immune reconstitution inflammatory syndrome, NA: not applicable, PML: progressive multifocal leukoencephalopathy

might actually be deleterious, as they can interfere with the appropriate control of JCV by decreasing the JCV-specific CD8+ T cells producing IFN- $\gamma$ and TNF $\alpha$. Glucocorticoids should be used to treat excessive and detrimental inflammation in the CNS (14). Of note, it is often difficult to distinguish PML with a controlled anti-viral inflammatory re- sponse from overwhelming PML-IRIS based on radiological findings alone. The ratio of CD4/CD8 obtained by a brain biopsy is useful for differentiating a regulated infectious immune response $(\mathrm{CD} 8+$ and $\mathrm{CD} 4+\mathrm{T}$ cells are comparable in balance) from fatal excessive inflammation (CD8+ $\mathrm{T}$ cells and numerous macrophages are dominant). Therefore, a 
brain biopsy is sometimes discussed in order to determine the $\mathrm{CD} 4 / \mathrm{CD} 8$ ratio in the affected regions before making a therapeutic decision concerning PML patients with gadolinium enhancement on brain MRI. The ratio of CD4/CD8 in the CSF might also reflect the immune condition and reactions against the infectious agent in the CNS (10). In our case, the clear effect of the first use of intravenous corticosteroid before the confirmed diagnosis of PML indicated that inflammation in the CNS was excessive rather than regulated (Fig. 3). Further studies are necessary to clarify the inflammatory state in PML lesions and indications of corticosteroid treatment without an invasive examination, such as a brain biopsy.

Patients with hematologic malignancy who underwent allogeneic hematopoietic stem cell transplantation (HSCT) have an elevated risk of developing PML due to their prolonged treatment with chemotherapy and immunosuppressive medications (15). Up to $40 \%$ of PML patients have hematological cancers, and the incidence rate of PML in patients with HSCT was estimated to be 35.4 in 100,000 personyears $(1,6)$. Although the cellular immune responses to JCV are activated after bone marrow transplantation, they cannot fully respond to JCV reactivation until 1 year after HSCT and increase 12 to 18 months after HSCT. In particular, patients with AML who are over 63 years old have difficulty generating JCV-specific cellular immune responses (16). PML can develop as early as 1.5 months after transplantation and is associated with the myeloablative conditioning regimen used to wipe out the HSCT recipient cells in preparation for transplantation (6). Our 61-year-old patient developed PML 2 years after HSCT and showed a low number of CD4+ T cells, so a poor prognosis was predicted. However, to our surprise, the patient's immune function was sufficiently preserved to cause excessive inflammation against JCV (Fig. 3).

In conclusion, we reported a case of hematologic malignancy-associated PML accompanied by recurrent episodes of inflammation in the CNS. Intermittent use of intravenous corticosteroids was effective for managing each exacerbation. Our case indicates that steroid pulse treatment should be considered as a therapeutic option in PML patients showing excessive inflammation, which is difficult to distinguish from controlled inflammatory responses based on neuroimaging findings alone.

The authors state that they have no Conflict of Interest (COI).

\section{References}

1. Mizusawa H, Kishida S, Saijo M, et al. [Progressive multifocal leukoencephalopathy (PML)]. Rinsho Shinkeigaku (Clin Neurol) 51: 1051-1057, 2011 (in Japanese, Abstract in English).

2. Wattjes MP, Wijburg MT, van Eijk J, et al. Inflammatory natalizumab-associated PML: baseline characteristics, lesion evolution and relation with PML-IRIS. J Neurol Neurosurg Psychiatry 89: 535-541, 2018.

3. Clifford DB, Yiannoutsos C, Glicksman M, et al. HAART improves prognosis in HIV-associated progressive multifocal leukoencephalopathy. Neurology 52: 623-625, 1999.

4. Clifford DB, De Luca A, DeLuca A, et al. Natalizumab-associated progressive multifocal leukoencephalopathy in patients with multiple sclerosis: lessons from 28 cases. Lancet Neurol 9: 438-446, 2010.

5. Neil EC, DeAngelis LM. Progressive multifocal leukoencephalopathy and hematologic malignancies: a single cancer center retrospective review. Blood Adv 1: 2041-2045, 2017.

6. Mateen FJ, Muralidharan R, Carone M, et al. Progressive multifocal leukoencephalopathy in transplant recipients. Ann Neurol 70: 305-322, 2011.

7. Sahraian MA, Radue EW, Eshaghi A, Besliu S, Minagar A. Progressive multifocal leukoencephalopathy: a review of the neuroimaging features and differential diagnosis. Eur J Neurol 19: 10601069, 2012.

8. Tan K, Roda R, Ostrow L, McArthur J, Nath A. PML-IRIS in patients with HIV infection: clinical manifestations and treatment with steroids. Neurology 72: 1458-1464, 2009.

9. Tan IL, McArthur JC, Clifford DB, Major EO, Nath A. Immune reconstitution inflammatory syndrome in natalizumab-associated PML. Neurology 77: 1061-1067, 2011.

10. Sanjo N, Kina S, Shishido-Hara Y, et al. Progressive multifocal leukoencephalopathy with balanced CD4/CD8 T-cell infiltration and good response to mefloquine treatment. Intern Med 55: 16311635, 2016.

11. Shelburne SA, Montes M, Hamill RJ. Immune reconstitution inflammatory syndrome: more answers, more questions. J Antimicrob Chemother 57: 167-170, 2006.

12. Gheuens S, Smith DR, Wang X, Alsop DC, Lenkinski RE, Koralnik IJ. Simultaneous PML-IRIS after discontinuation of natalizumab in a patient with MS. Neurology 78: 1390-1393, 2012.

13. Vermersch P, Kappos L, Gold R, et al. Clinical outcomes of natalizumab-associated progressive multifocal leukoencephalopathy. Neurology 76: 1697-1704, 2011.

14. Antoniol C, Jilek S, Schluep M, et al. Impairment of JCV-specific T-cell response by corticotherapy: effect on PML-IRIS management? Neurology 79: 2258-2264, 2012.

15. Yuan C, Deberardinis C, Patel R, et al. Progressive multifocal leukoencephalopathy after allogeneic stem cell transplantation: Case report and review of the literature. Transpl Infect Dis 20: e12879, 2018.

16. Tan CS, Broge TA, Ngo L, et al. Immune reconstitution after allogeneic hematopoietic stem cell transplantation is associated with selective control of JC virus reactivation. Biol Blood Marrow Transplant 20: 992-999, 2014.

The Internal Medicine is an Open Access journal distributed under the Creative Commons Attribution-NonCommercial-NoDerivatives 4.0 International License. To view the details of this license, please visit (https://creativecommons.org/licenses/ by-nc-nd/4.0/).

(C) 2021 The Japanese Society of Internal Medicine Intern Med 60: 1287-1291, 2021 